\title{
Resting-state network topology and planning ability in healthy adults
}

\author{
Chris Vriend ${ }^{1,2,3} \cdot$ Margot J. Wagenmakers $^{1} \cdot$ Odile A. van den Heuvel $^{1,2} \cdot$ Ysbrand D. van der Werf $^{1}$
}

Received: 11 July 2019 / Accepted: 6 December 2019 / Published online: 21 December 2019

(c) The Author(s) 2019

\begin{abstract}
Functional magnetic resonance imaging (fMRI) studies have been used extensively to investigate the brain areas that are recruited during the Tower of London (ToL) task. Nevertheless, little research has been devoted to study the neural correlates of the ToL task using a network approach. Here we investigated the association between functional connectivity and network topology during resting-state fMRI and ToL task performance, that was performed outside the scanner. Sixty-two (62) healthy subjects (21-74 years) underwent eyes-closed rsfMRI and performed the task on a laptop. We studied global (whole-brain) and within subnetwork resting-state topology as well as functional connectivity between subnetworks, with a focus on the default-mode, fronto-parietal and dorsal and ventral attention networks. Efficiency and clustering coefficient were calculated to measure network integration and segregation, respectively, at both the global and subnetwork level. Our main finding was that higher global efficiency was associated with slower performance $\left(\beta=0.22, P_{\mathrm{bca}}=0.04\right)$ and this association seemed mainly driven by inter-individual differences in default-mode network connectivity. The reported results were independent of age, sex, education-level and motion. Although this finding is contrary to earlier findings on general cognition, we tentatively hypothesize that the reported association may indicate that individuals with a more integrated brain during the resting-state are less able to further increase network efficiency when transitioning from a rest to task state, leading to slower responses. This study also adds to a growing body of literature supporting a central role for the default-mode network in individual differences in cognitive performance.
\end{abstract}

Keywords Functional connectivity $\cdot$ Resting-state $\cdot$ Network analysis $\cdot$ Planning $\cdot$ Cognition $\cdot$ Default-mode network

\section{Introduction}

Executive functions are a set of mental processes that enable us to plan, focus attention, remember instructions and handle several tasks at once (Diamond 2013). Functional magnetic

Electronic supplementary material The online version of this article (https://doi.org/10.1007/s00429-019-02004-6) contains supplementary material, which is available to authorized users.

Chris Vriend

c.vriend@amsterdamumc.nl

1 Department of Anatomy and Neurosciences, Amsterdam UMC, Vrije Universiteit Amsterdam, Amsterdam Neuroscience, De Boelelaan 1117, Amsterdam, The Netherlands

2 Department of Psychiatry, Amsterdam UMC, Vrije Universiteit Amsterdam, Amsterdam Neuroscience, De Boelelaan 1117, Amsterdam, The Netherlands

3 Department of Anatomy and Neuroscience, Amsterdam UMC, Location VUmc, p/a sec. ANW Ol2, BT, PO Box 7007, 1007 MB Amsterdam, The Netherlands resonance imaging (fMRI) studies have shown that these functions are associated with functional connectivity (FC) of certain resting-state networks (RSN) (Rabinovici et al. 2015; Funahashi and Andreau 2013; Nowrangi et al. 2014). Various RSN have been shown to be involved in executive functions, including the default mode network (DMN) which is active during rest and deactivates during task performance (Buckner et al. 2008; Mak et al. 2017; Anticevic et al. 2012). Other relevant RSN for cognition are the frontoparietal network (FPN) (Cole et al. 2012, 2014b) and the dorsal and ventral attention networks (DAN and VAN, respectively) (Fortenbaugh et al. 2018). Although the utility of RSN in cognitive neuroscience and understanding of the neural correlates of cognition has been debated (Campbell and Schacter 2017; Davis et al. 2017; Iordan and Reuter-Lorenz 2017), resting-state FC patterns show good correspondence with task-based FC patterns (Krienen et al. 2014), are fundamentally stable (Gratton et al. 2018) and may act as an intrinsic network architecture that shapes FC when evoked by a cognitive task (Cole et al. 2014a; Ito et al. 2017). 
The architecture or topology of the brain can be studied using graph analysis, where the brain is simplified to a graph of nodes (i.e., different brain regions) and edges (i.e., connections between brain regions) (Wang et al. 2010; Bullmore and Sporns 2009). Different properties of the brain network can be calculated using this graph. For example, efficiency and clustering describe the ability of a network to integrate and segregate information, respectively (Cohen and D'Esposito 2016; Lord et al. 2017). The brain balances its ability to integrate and easily transmit information throughout the network, and to segregate information processing in clusters of highly interconnected (specialized) neighboring nodes (Bullmore and Sporns 2009). This ability of the brain for integration and segregation is vital for cognitive processes (Cohen and D'Esposito 2016) and higher intelligence has been associated with a more efficient network topology (Langer et al. 2012; van den Heuvel et al. 2009). Conversely, dementia and cognitive impairments in the light of brain disorders generally show dysfunction in the brain's ability to functionally integrate and segregate information (Dai et al. 2019; Lopes et al. 2017; Rocca et al. 2016). Nevertheless, studies on the associations between network topology and inter-individual differences in cognitive functions in healthy subjects are relatively scarce, (e.g., Cohen and D'Esposito 2016; Sheffield et al. 2017), and to the best of our knowledge, no study has yet focused on the association between network topology and planning capacity. Planning is the ability to think ahead to achieve a goal via a series of intermediate steps (Owen 1997) and is a vital function in daily life that we here operationalize in the form of the Tower of London (ToL) task. In this study, we investigated the association between RSN topology and planning performance, using a graph-based approach. Based on prior research (Langer et al. 2012; van den Heuvel et al. 2009; Sheffield et al. 2017), we hypothesized a positive relationship between network topology measured during restingstate and cognitive planning ability, measured using the ToL task performed outside of the scanner.

\section{Methods}

\section{Subjects and measurements}

Data of healthy adult controls from two previous case-control studies (Gerrits et al. 2015; de Wit et al. 2012) were pooled for the current study. Exclusion criteria for all healthy subjects were the use of psychoactive medication, current or past psychiatric diagnosis, a history of a major physical or neurological illness, MRI contraindications or a history of alcohol abuse. Further exclusion criteria for the current study were: no available data on the ToL task, extreme behavioral scores ( $\geq 2 \mathrm{SD}$ from the mean), a time-interval of more than 21 days between resting-state fMRI (rs-fMRI) and performing the ToL task, or pathological incidental findings on the structural MRI scan. Written informed consent was provided by all participants according to the Declaration of Helsinki and the studies were approved by the Medical Ethical Committee of the VU University Medical Centre (Amsterdam, The Netherlands).

The participants performed a computerized version of the ToL task as a measure of planning (Phillips et al. 2001; Shallice 1982). Details of the ToL task are provided in the study by (van den Heuvel et al. 2003). In short, the participants saw two configurations ("begin" and "goal" position) of three colored beads on vertical posts of different heights. The purpose of the task is to determine the minimum number of moves $(1,2,3,4$, or 5$)$ needed to match the configuration of the goal position. Participants responded via the matching keyboard-button. The first post can hold all three beads, the second two, and the third post one. Only one bead can be moved at a time and only if there is no other bead on top of it. Prior to the experiment, participants were provided verbal and written explanation and performed a practice run. Performance on the ToL task was indicated by the mean accuracy and mean reaction time on correct trials across all five difficulty levels (Kaller et al. 2016). Intelligence scores were approximated by the Dutch Adult Reading test (NLV; (Schmand et al. 1991). We scored education level according to the Dutch Verhage scale (Verhage 1964) that ranges from 1-primary school not finished, to 7-university or higher. Handedness was assessed using the Edinburgh Handedness Inventory (Oldfield 1971).

\section{MR image acquisition}

MR images were acquired at Amsterdam UMC, location VUmc (Amsterdam, The Netherlands) on a GE Signa HDxt 3 T MRI scanner (General Electric, Milwaukee, WI, USA) with an eight-channel head coil. The participant's head was immobilized using foam pads to reduce motion artifacts. Participants were told to lie still, keep their eyes closed and not fall asleep during the acquisition of the rs-fMRI scan (duration: $5.9 \mathrm{~min}$ ). T2*-weighted echo-planar (EPI) images were acquired with $\mathrm{TR}=1.8 \mathrm{~s}$, TE $=35 \mathrm{~ms}, 64 \times 64$ matrix, field of view $=24 \mathrm{~cm}$ and flip angle $=80^{\circ}$ and 40 ascending slices per volume $(3.75 \times 3.75 \mathrm{~mm}$ in plane resolution; slice thickness $=2.8 \mathrm{~mm}$; interslice gap $=0.2 \mathrm{~mm}$ ). Structural scanning encompassed a sagittal three-dimensional gradient-echo T1-weighted sequence $(256 \times 256$ matrix; voxel size $=1 \times 0.977 \times 0.977 \mathrm{~mm}$; 172 slices $)$.

\section{Image (pre)processing}

RS-fMRI and T1-weighted images were preprocessed with FMRIB's Software Library version 5.0.10 (FSL; (Smith 
et al. 2004)). The first four volumes were discarded to reach steady-state magnetization. Non-brain tissue was removed using BET and the structural image was segmented into gray (GM), white matter (WM) and cerebrospinal fluid (CSF) using FAST. Functional images were re-aligned using McFLIRT and the resulting six rigid-body parameters were used to calculate the motion parameters. Functional images were spatially smoothed with a $5 \mathrm{~mm}$ full width at half maximum (FWHM) kernel. Subjects with significant motion during scanning, defined as a mean relative root mean squared displacement $(\mathrm{RMS})>0.2 \mathrm{~mm}$, or $>20$ volumes with framewise relative RMS displacement $>0.25 \mathrm{~mm}$, were excluded (Ciric et al. 2017). Because rs-fMRI is exceptionally sensitive to motion artefacts (Power et al. 2015), we additionally performed ICA-AROMA (Pruim et al. 2015). ICA-AROMA is a single-subject denoising strategy based on independent component analysis (ICA) that automatically identifies motion-related components in the functional data based on their high-frequency content, correlation with the motion parameters and edge and CSF fraction and removes their variance from the data (Pruim et al. 2015). ICA-AROMA has been shown to provide a good trade-off between reducing noise and preserving BOLD signal (Ciric et al. 2017; Pruim et al. 2015; Parkes et al. 2018). After ICA-AROMA, additional nuisance regression was performed by removing signal from the WM and CSF and functional images were high-pass filtered (100 s cut-off).

The functional scan was registered to the anatomical T1-scans using boundary-based registration (FSL epi_reg). The anatomical image was parcellated into 225 nodes; 210 cortical nodes were defined based on the Brainnetome Atlas (Fan et al. 2016), 14 subcortical nodes were individually segmented using FSL FIRST (Patenaude et al. 2011) and one cerebellar node was defined based on the FSL's cerebellar atlas (Diedrichsen et al. 2009). EPI distortions during fMRI can lead to signal drop-out. To account for signal dropout near air/tissue boundaries during scanning, we applied a mask to the functional scan to exclude voxels with signal intensities in the lowest quartile of the robust range (Meijer et al. 2017). Nodes were discarded if they comprised less than four signal-containing voxels. This rendered a total of 194 common brain regions across all subjects. Time-series were extracted from each node. The cortical nodes were subdivided into four RSN: the DMN, FPN, DAN and VAN based on the functional subdivision by Yeo et al. (2011); see supplementary Table 1.

\section{Functional connectivity matrices}

To measure FC and construct connectivity matrices we applied wavelet coherence on the time-series of each possible pair of the 194 brain regions within the frequency range 0.06 and $0.12 \mathrm{~Hz}$ (Chang and Glover 2010). Wavelet coherence has several advantages over Pearson's correlations, including denoising properties and robustness to outliers (Gu et al. 2017; Fadili and Bullmore 2004; Achard et al. 2006). The $0.06-0.12 \mathrm{~Hz}$ frequency range was chosen because it has been suggested to be a reliable and robust range that is associated with cognitive performance (Zhang et al. 2016; Bassett et al. 2013). We applied wavelet coherence to the entire rs-fMRI scan to calculate the network measures (see below). An overview of the (pre)processing pipeline is provided in Fig. 1.

\section{Network measures}

At the global level, we calculated global efficiency and global clustering coefficient (Gcc). Global efficiency is the inverse of the average path length (i.e., the maximum connectivity between each pair of nodes), with high efficiency meaning that information can rapidly travel through the whole network (Latora and Marchiori 2001). Gcc is equivalent to the proportion of the actual number of edges between the nearest neighbors of a node to all possible edges and signifies the tendency of the whole network to segregate into locally interconnected triplets that function as a specialized subunit (Rubinov and Sporns 2010). Test-retest reliability of global efficiency and Gcc are fair-to-good (Welton et al. 2015). At the subnetwork level, we calculated efficiency and clustering coefficient for each of the four RSNs (DMN, FPN, DAN and VAN). In addition, we determined the mean FC between each of the four RSNs (resulting in six betweennetwork mean FC values). Test-retest reliability of these measures at the subnetwork level is unknown.

\section{Data analysis}

Statistical analyses were performed using SPSS version 25 (IBM Corp, Armonk, NY, USA). We describe demographical characteristics and performance on the ToL task using means and standard deviations unless indicated otherwise. Pearson's $(r)$ or Spearman's rho $\left(r_{\mathrm{s}}\right)$ correlations were performed between demographic and performance measures, depending on the distribution. We performed bootstrapped hierarchal multiple regression analysis to investigate the association between network measures (predictors) and accuracy and reaction time on the ToL task (outcome measures). Because age was correlated with performance, age was entered in the first block of all models. The network measure of interest and mean RMS displacement, as a measure for motion, were entered in the second and third block, respectively. As a sensitivity analysis, we entered sex or education level to the fourth block of the model. The regression models were bootstrapped using 2000 iterations. We report bias and accelerated (BCa) confidence intervals and the accompanying $P$ values $\left(P_{\mathrm{bca}}\right)$ as they account for bias and 


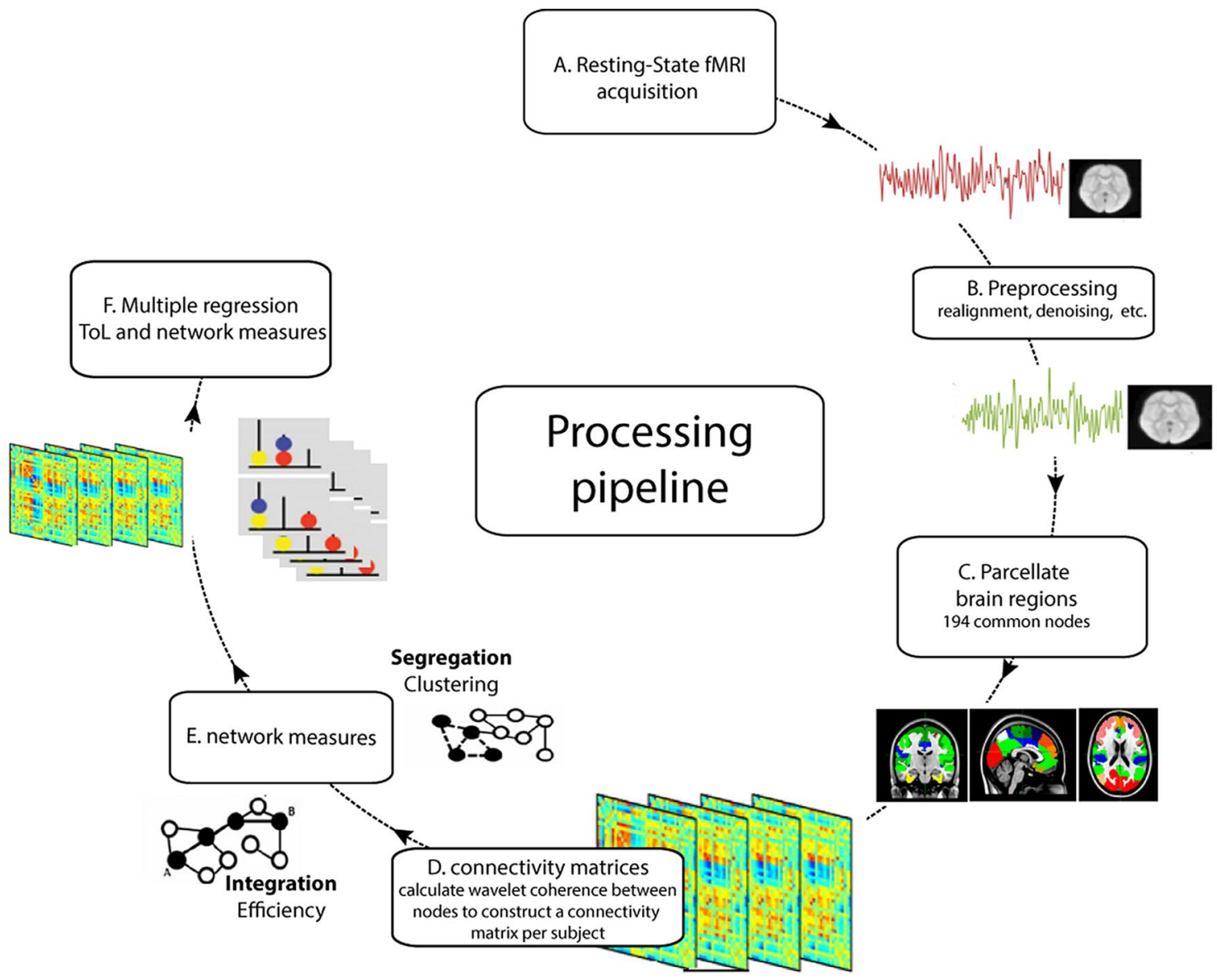

Fig. 1 Outline of the processing pipeline. (A) Resting-state fMRI data were collected and (B) pre-processed. The brain was (C) parcellated into separate brain regions (nodes). There were 194 nodes common to all subjects with enough signal to (D) construct connectivity matrices (see text) using wavelet coherence. (E) network measures were calculated from each connectivity matrix on the global and subnetwork level. (F) multiple regression analyses were applied to relate performance on the Tower of London (ToL) task to network measures skewness in the data and provide a more robust estimate of the association that is less reliant on the distribution of the variable. All assumptions of multiple regression analyses, including homoscedasticity of residuals, were assessed and met. We performed separate analyses for the network measures on the global level and on the subnetwork level. On the subnetwork level, type I errors due to multiple comparisons were minimized using the False Discovery Rate [FDR, $q<0.05$ (Benjamini and Hochberg 1995)]. Statistical significance was set to $P<0.05$ for all analyses. No formal power analysis was conducted prior to the execution of this study.

\section{Results}

\section{Sample characteristics and behavioral results}

Of the 69 participants with an available ToL task and rsfMRI data, seven had to be excluded (see Fig. 2), which

\section{Flow Chart}

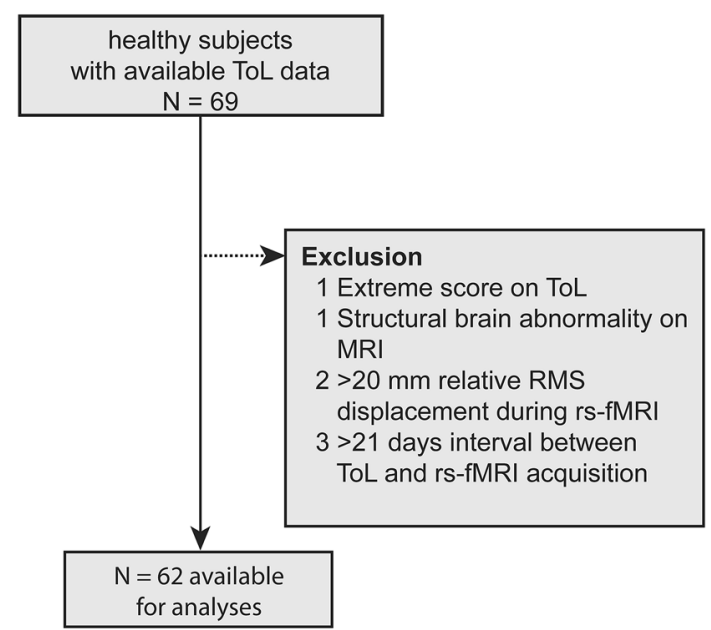

Fig. 2 Flowchart of participant exclusion 
resulted in a total sample size of 62 participants, aged between 21 and 74 years old $\left(M_{\text {age }}=48.1 \pm 13.9,33\right.$ males $)$. The time between performing the ToL task and the rsfMRI was on average $6.2 \pm 4.6$ (range 0-21) days. See Table 1 for the sample characteristics. Age showed a positive correlation with reaction time $(r=0.498, P<0.001)$ but only a trend-level negative correlation with accuracy ( $r=-0.243, P=0.057$ ) indicating that older participants tended to respond slower and slightly less accurately. The average motion during rs-fMRI (expressed as mean relative RMS framewise displacement) was $0.068 \pm 0.029$ (range $0.027-0.17$ ) and was positively correlated with age $\left(r_{\mathrm{s}}=0.34, P=0.007\right)$ but not performance on the ToL (reaction time: $r_{\mathrm{s}}=0.06, P=0.66$; accuracy: $r_{\mathrm{s}}=-0.18$, $P=0.12)$.

Table 1 Sample characteristics

\begin{tabular}{ll}
\hline$N$ subjects $(\%$ female) & $62(46.8)$ \\
Age (years) & $48.1 \pm 13.9$ \\
Education level (in \%) $^{\mathrm{a}}$ & \\
3 & 1.6 \\
4 & 48 \\
5 & 29.0 \\
6 & 43.5 \\
7 & 17.7 \\
Handedness (R/L) & $54 / 7$ \\
ToL accuracy $(\%)$ & $87.7 \pm 7.5$ \\
ToL reaction time (s) & $10.1 \pm 2.1$ \\
Mean relative RMS & $0.07 \pm 0.03$ \\
\hline
\end{tabular}

${ }^{\mathrm{a}}$ Missing for two subjects

${ }^{\mathrm{b}}$ Missing for one subject

\section{Global topology}

Global efficiency $\left(\beta=0.22, P_{\mathrm{bca}}=0.04\right)$ but not Gcc $\left(\beta=-0.09, P_{\mathrm{bca}}=0.57\right)$ was positively associated with reaction time above and beyond the effects of age (see Table 2 and Fig. 3). There were no significant associations with accuracy. Adding sex or education level as a nuisance covariate to the model had no effect on these results.

\section{Subnetwork topology}

Both efficiency and clustering of the DMN (efficiency: $\beta=0.25, P_{\mathrm{bca}}=0.018$; clustering: $\beta=0.23, P_{\mathrm{bca}}=0.039$ ) but not the other subnetworks (see supplemental Table 2) were positively related to reaction time. These associations did not, however, survive the multiple comparison correction (DMN efficiency $P_{\mathrm{fdr}}=0.072$; DMN clustering: $\left.P_{\text {fdr }}=0.077\right)$. Adding sex or education level as additional nuisance covariate to the model had no effect on the results. Consistent with the results on the global level, there were no significant associations with the accuracy of task performance (Supplemental Table 3).

\section{Between-subnetwork connectivity}

FC between the DMN and FPN $\left(\beta=0.23, P_{\mathrm{bca}}=0.04\right)$, the DAN $\left(\beta=0.21, P_{\text {bca }}=0.04\right)$ and the VAN $(\beta=0.20$, $\left.P_{\text {bca }}=0.04\right)$ were all positively associated with reaction time. These associations did not survive the FDR correction for multiple comparisons (all $P_{\mathrm{fdr}}=0.09$; supplemental Tables 4 and 5).
Table 2 Associations between TOL performance and global network measures

\begin{tabular}{lllllll}
\hline TOL & Model & $B(\mathrm{SE})$ & $95 \% \mathrm{CI}(\mathrm{BCa})$ & Beta & $P_{\mathrm{bCa}}$ & $R^{2}$ \\
\hline RT & Age & $0.09(0.015)$ & $0.06,0.12$ & 0.595 & $<0.001$ & \\
& GE & $9.79(4.72)$ & $0.84,19.8$ & 0.219 & 0.039 & 0.293 \\
& Motion & $-16.46(6.53)$ & $-30.6,-5.7$ & -0.230 & 0.008 & \\
& Age & $0.08(0.017)$ & $0.04,0.11$ & 0.531 & $<0.001$ & \\
& Gcc & $-32.88(53.72)$ & $-133.9,68.8$ & -0.089 & 0.567 & 0.252 \\
& Motion & $-12.03(7.69)$ & $-26.6,2.3$ & -0.168 & 0.113 & \\
ACC & Age & $-0.131(.073)$ & $-0.28,-0.009$ & -0.241 & 0.079 & \\
& GE & $-31.1(21.57)$ & $-76.3,9.6$ & -0.194 & 0.156 & 0.06 \\
& Motion & $-21.62(29.23)$ & $-69.0,43.1$ & -0.084 & 0.450 & \\
& Age & $-0.09(.075)$ & $-0.24,0.05$ & -0.164 & 0.249 & \\
& Gcc & $206.28(155.46)$ & $-79.3,515.3$ & 0.154 & 0.187 & 0.05 \\
& Motion & $-41.39(27.02)$ & $-89.5,13.3$ & -0.161 & 0.117 & \\
\hline
\end{tabular}

For each analysis, age was entered in model 1, the network measure in model 2 and motion parameters in model 3. Only the results of model 3 are shown here. $P$ values are bootstrapped using 2000 permutations

$T O L$ Tower of London task, $R T$ reaction time, ACC accuracy, $S E$ standard error, $C I$ confidence interval, $B C a$ Bias corrected and accelerated, $G E$ global efficiency, $G c c$ global clustering coefficient 


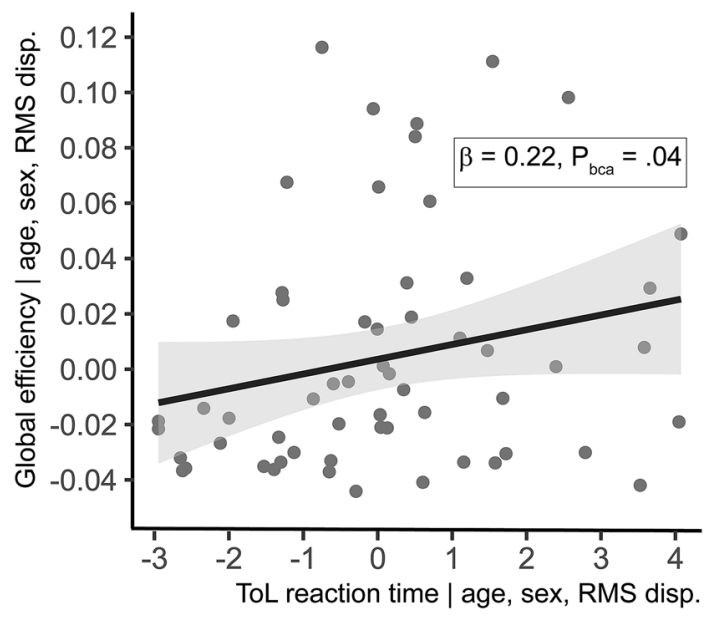

Fig. 3 Partial correlation plot of the association between reaction time on the Tower of London task and Global (whole-brain) efficiency. ToL Tower of London, RMS disp. mean root-mean-squared framewise displacement

\section{Post-hoc analyses}

Because of possible floor/ceiling effects during the less demanding 1, 2 and 3 step trials of the ToL task, we re-ran the regression models using only the mean accuracy rates and reaction times during ToL steps 4 and 5. These post-hoc analyses showed that at the global level reaction time-but not accuracy - was still associated with global efficiency $\left(\beta=0.27, P_{\mathrm{bca}}=0.04\right)$, not Gcc $\left(\beta=-0.09, P_{\mathrm{bca}}=0.59\right)$. At the subnetwork level, efficiency of the DMN $(\beta=0.41$, $\left.P_{\mathrm{fdr}}=0.001\right)$ and FPN $\left(\beta=0.32, P_{\mathrm{fdr}}=0.045\right)$, clustering of the DMN $\left(\beta=0.33, P_{\text {fdr }}=0.05\right)$ and FC between the DMN and FPN $\left(\beta=0.38, P_{\mathrm{fdr}}=0.02\right)$ were all positively associated with reaction time, after FDR correction for multiple comparisons.

\section{Discussion}

In this study in 62 healthy adults with a wide age range we investigated the association between network topology during a rs-fMRI session and cognitive planning ability during a ToL task that was performed outside the scanner. We observed that global (whole-brain) efficiency was associated with reduced planning speed and that this effect was mainly driven by the FC of the DMN. The results were independent from inter-individual differences in age, gender, education level and motion during rs-fMRI. Post-hoc analyses showed that our results were strongest when focusing on the higher task load trials of the ToL task (four and five step trials).

Global efficiency provides a measure of how well-integrated a network is and how easily information can travel from one node to another on the other side of the network, while the clustering coefficient is a measure of how wellconnected nodes are locally into segregated triangles of neighboring nodes. Both measures are often used to describe the characteristics of a network and abnormalities in these network measures are commonly observed in the structural and functional networks of patients with a brain disorder (Bullmore and Sporns 2012; Griffa et al. 2013; Worbe 2015; Lord et al. 2017). Here we observed that subjects with a higher global efficiency show slower planning performance on the ToL task. This finding is at odds with our hypothesis and previous studies that observed that higher global efficiency is associated with higher global intelligence (van den Heuvel et al. 2009; Sheffield et al. 2017) and performance on working memory tasks (Cohen and D'Esposito 2016; Sheffield et al. 2015). One other study has also previously found that a higher global efficiency was associated with worse performance on a working memory task, but only in older adults and only when focusing on task-based FC (Stanley et al. 2015). This is the first study, however, to investigate planning ability. One possible, albeit less plausible, explanation might, therefore, be that planning requires a different whole-brain network organization than working memory tasks or general intelligence. Alternatively, the higher global efficiency in individuals with slower performance on the ToL task may also point towards a more random network (Ajilore et al. 2014). As there was no association between ToL task speed and lower global clustering (a characteristic feature of random networks), this explanation is also less viable.

Studies have shown that, although the resting-state provides a core and intrinsic network architecture that highly overlaps with the network topology of task-states (Cole et al. 2010; Krienen et al. 2014), significant reorganization does take place during the execution of tasks, and the magnitude and spatial redistribution depends on the task and its load (Cohen and D'Esposito 2016; Davison et al. 2015). Furthermore, the ease with which a network can reconfigure from rest to task-states correlates with task performance and general cognition (Braun et al. 2015; Bassett et al. 2011; Telesford et al. 2016; Hearne et al. 2017). Transitions of rest to (demanding) task-states have generally been associated with an increase in global efficiency, signifying a better integrated network (Cohen and D'Esposito 2016; Hearne et al. 2017; Shine et al. 2016; see Shine and Poldrack 2018 for a review). This increase in network integration is, however, not unconstrained, as a fully integrated functional network would lead to epileptic seizures and violates the principles of cost-efficiency (Shine and Poldrack 2018; Bullmore and Sporns 2012). Assuming that in our subjects network integration would similarly increase from the resting-state to task-state, i.e., execution of the ToL task, it is conceivable that global efficiency could not increase sufficiently in those subjects with an already highly integrated network during 
the resting-state to meet task demands, leading to a slower behavioral response. This concept is schematically depicted in Fig. 4. Although this hypothesis receives indirect support from multiple previous studies on dynamic network reconfigurations (Shine and Poldrack 2018), we unfortunately did not acquire fMRI scans during the execution of the ToL task and, therefore, this explanation currently remains speculative. Because the slower responses were not associated with lower accuracy $\left(r_{\mathrm{s}}=-0.19, P=0.13\right)$ and we did not observe an association between network topology and accuracy, our results may not be specific for planning performance but may also be related to an overall slower information processing speed. Why we did not find an association with task accuracy is currently unclear.

At the subnetwork level, we showed that our global results were mainly driven by inter-individual differences in $\mathrm{FC}$ of the DMN; both the topology of the DMN and FC between the DMN and the other RSNs (mainly the FPN) were associated with slower task performance. Because closer inspection showed that efficiency and clustering of the DMN were highly correlated $(r=0.84)$, the observed positive associations should instead be interpreted as an association between slower performance and increased within DMN FC. Indeed, when looking at total FC within the DMN, we observed a positive association $\left(\beta=0.25, P_{\mathrm{bca}}=0.02\right)$ with ToL reaction time. It is generally accepted that activity within the DMN is high when a subject is not engaged in any specific task and its activity is suppressed when external stimuli demand cognitive engagement (Anticevic et al. 2012). Heightened DMN activity and higher FC between the DMN and other RSNs are also commonly associated with reduced cognitive performance in brain disorder-related deficits (Putcha et al. 2016; Esposito et al. 2018; Anticevic et al. 2012). Our associations

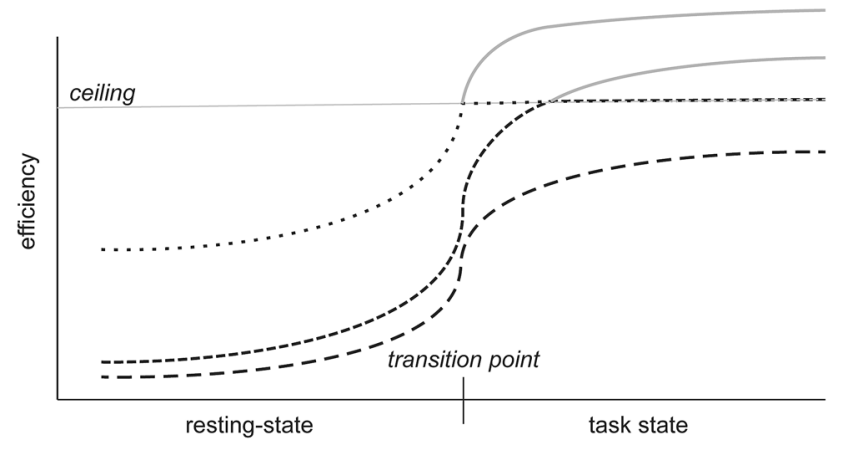

Fig. 4 Schematic representation of rest-to-task reconfiguration hypothesis. The figure shows three fictional subjects that transition from a resting-state to task state and show a concomitant increase in (global) efficiency. The top two subjects, already have such a high efficiency during resting-state that when the brain network needs to reconfigure to a more integrated state to meet task demands, efficiency cannot surpass the ceiling (horizontal dotted lines) and leads to slower responses between slower ToL performance and increased within DMN FC and increased connectivity between the DMN and the other RSNs is therefore in line with these findings and adds to the growing body of literature that shows that inter-individual differences in FC of the DMN is associated with cognitive performance, even in normally functioning healthy subjects. It must be noted that these associations did not survive the multiple comparison correction, although the reported associations between performance speed at higher task load and within DMN FC and FC between DMN and FPN in our post-hoc analysis did pass the FDR correction.

In recent years, scientific awareness has increased for the low reproducibility of neuroimaging findings (Nichols et al. 2017). Test-retest reliability is often used as a measure for reproducibility and generalizability. Although graph measures, such as global efficiency and Gcc, show fair-to-good test-retest reliability (Welton et al. 2015), a recent metaanalysis showed that edges within a functional connectivity matrix - on the basis of which graph measures are calculated-show poor test-retest reliability (Noble et al. 2019). This low test-retest reliability influences statistical power and necessitates the inclusion of larger samples to reach the effect size of interest (Matheson 2019; Zuo et al. 2019). It is, however, important to note that test-retest reliability is not the same as validity and the meta-analysis showed that one of the main factors that influenced test-retest reliability was artefact correction (Noble et al. 2019); a necessary step during preprocessing to remove motion and other non-neural physiological noise from the data and avoid spurious results (Parkes et al. 2018). Moreover, although absolute values of intra-individual edges show low reproducibility (Noble et al. 2019), inter-individual differences in the functional connectome are stable across, days, months and even years (Horien et al. 2019; Finn et al. 2015; Miranda-Dominguez et al. 2014), and its characteristics are uniquely associated with a particular individual across time (Horien et al. 2019). This provides justification for predicting a person's phenotype, including cognitive functioning, on the basis of betweensubject variability of the functional connectome. Another part of reproducibility is transparent and complete reporting of the methods and results. To that end, we report the COBIDAS checklist in the supplementary material (Nichols et al. 2017). We will make the here reported data available to researchers upon reasonable request.

A limitation of this study is that we exclusively looked at resting-state FC to predict performance on the ToL and not-at task-based FC, i.e. during the execution of the ToL itself. This would have allowed us to look directly at the network characteristics associated with performance and to test our hypothesis of reduced ability to network integration when transitioning from rest to task. Furthermore, although conscious state may alter network topology, we did not include an objective measure to ensure wakefulness 
during the eyes-closed resting-state scan. A strength of this study is that we retrospectively recruited a relatively large number of healthy subjects and used stringent control for (micro)motion by excluding subjects with $>0.2 \mathrm{~mm}$ mean RMS displacement, denoising rs-fMRI for motion-related artifacts with ICA-AROMA, employing wavelet coherence to construct the connectivity matrices and adding RMS displacement to the regression model.

In conclusion, we showed that higher global efficiency during rest and higher FC of the DMN with other RSNs and within itself is associated with slower planning performance. We tentatively postulate that due to ceiling effects individuals with a higher integrative network state during rest are less able to reconfigure to a more integrated state during task execution, leading to slower exchange across the brain network and slower behavioral responses.

Acknowledgements The authors want to acknowledge Dr. Niels Gerrits and Dr. Stella de Wit for carrying out the data collection.

\section{Compliance with ethical standards}

Conflict of interest The authors have nothing to declare.

Open Access This article is licensed under a Creative Commons Attribution 4.0 International License, which permits use, sharing, adaptation, distribution and reproduction in any medium or format, as long as you give appropriate credit to the original author(s) and the source, provide a link to the Creative Commons licence, and indicate if changes were made. The images or other third party material in this article are included in the article's Creative Commons licence, unless indicated otherwise in a credit line to the material. If material is not included in the article's Creative Commons licence and your intended use is not permitted by statutory regulation or exceeds the permitted use, you will need to obtain permission directly from the copyright holder. To view a copy of this licence, visit http://creativecommons.org/licenses/by/4.0/.

\section{References}

Achard S, Salvador R, Whitcher B, Suckling J, Bullmore E (2006) A resilient, low-frequency, small-world human brain functional network with highly connected association cortical hubs. J Neurosci 26(1):63-72. https://doi.org/10.1523/JNEUROSCI.3874-05.2006

Ajilore O, Lamar M, Kumar A (2014) Association of brain network efficiency with aging, depression, and cognition. Am J Geriatr Psychiatry 22(2):102-110. https://doi.org/10.1016/j. jagp.2013.10.004

Anticevic A, Cole MW, Murray JD, Corlett PR, Wang XJ, Krystal JH (2012) The role of default network deactivation in cognition and disease. Trends Cogn Sci 16(12):584-592. https://doi. org/10.1016/j.tics.2012.10.008

Bassett DS, Wymbs NF, Porter MA, Mucha PJ, Carlson JM, Grafton ST (2011) Dynamic reconfiguration of human brain networks during learning. Proc Natl Acad Sci USA 108(18):7641-7646. https:// doi.org/10.1073/pnas.1018985108

Bassett DS, Wymbs NF, Rombach MP, Porter MA, Mucha PJ, Grafton ST (2013) Task-based core-periphery organization of human brain dynamics. PLoS Comput Biol 9(9):e1003171. https://doi. org/10.1371/journal.pcbi.1003171

Benjamini Y, Hochberg Y (1995) Controlling the false discovery ratea practical and powerful approach to multiple testing. J R Stat Soc Ser B Stat Methodol 57(1):289-300

Braun U, Schafer A, Walter H, Erk S, Romanczuk-Seiferth N, Haddad L, Schweiger JI, Grimm O, Heinz A, Tost H, Meyer-Lindenberg A, Bassett DS (2015) Dynamic reconfiguration of frontal brain networks during executive cognition in humans. Proc Natl Acad Sci USA 112(37):11678-11683. https://doi.org/10.1073/ pnas. 1422487112

Buckner RL, Andrews-Hanna JR, Schacter DL (2008) The brain's default network: anatomy, function, and relevance to disease. Ann N Y Acad Sci 1124:1-38. https://doi.org/10.1196/annals.1440.011

Bullmore E, Sporns O (2009) Complex brain networks: graph theoretical analysis of structural and functional systems. Nat Rev Neurosci 10(3):186-198. https://doi.org/10.1038/nrn2575

Bullmore E, Sporns O (2012) The economy of brain network organization. Nat Rev Neurosci 13(5):336-349. https://doi. org/10.1038/nrn3214

Campbell KL, Schacter DL (2017) Aging and the resting state: is cognition obsolete? Lang Cogn Neurosci 32(6):661-668. https ://doi.org/10.1080/23273798.2016.1227858

Chang C, Glover GH (2010) Time-frequency dynamics of restingstate brain connectivity measured with fMRI. Neuroimage 50(1):81-98. https://doi.org/10.1016/j.neuroimage.2009.12.011

Ciric R, Wolf DH, Power JD, Roalf DR, Baum GL, Ruparel K, Shinohara RT, Elliott MA, Eickhoff SB, Davatzikos C, Gur RC, Gur RE, Bassett DS, Satterthwaite TD (2017) Benchmarking of participant-level confound regression strategies for the control of motion artifact in studies of functional connectivity. Neuroimage 154:174-187. https://doi.org/10.1016/j.neuroimage .2017 .03 .020

Cohen JR, D'Esposito M (2016) The segregation and integration of distinct brain networks and their relationship to cognition. J Neurosci 36(48):12083-12094. https://doi.org/10.1523/JNEUR OSCI.2965-15.2016

Cole DM, Smith SM, Beckmann CF (2010) Advances and pitfalls in the analysis and interpretation of resting-state FMRI data. Front Syst Neurosci 4:8. https://doi.org/10.3389/fnsys.2010.00008

Cole MW, Yarkoni T, Repovs G, Anticevic A, Braver TS (2012) Global connectivity of prefrontal cortex predicts cognitive control and intelligence. J Neurosci 32(26):8988-8999. https://doi. org/10.1523/JNEUROSCI.0536-12.2012

Cole MW, Bassett DS, Power JD, Braver TS, Petersen SE (2014a) Intrinsic and task-evoked network architectures of the human brain. Neuron 83(1):238-251. https://doi.org/10.1016/j.neuro n.2014.05.014

Cole MW, Repovs G, Anticevic A (2014b) The frontoparietal control system: a central role in mental health. Neuroscientist 20(6):652664. https://doi.org/10.1177/1073858414525995

Dai Z, Lin Q, Li T, Wang X, Yuan H, Yu X, He Y, Wang H (2019) Disrupted structural and functional brain networks in Alzheimer's disease. Neurobiol Aging 75:71-82. https://doi.org/10.1016/j. neurobiolaging.2018.11.005

Davis SW, Stanley ML, Moscovitch M, Cabeza R (2017) Resting-state networks do not determine cognitive function networks: a commentary on Campbell and Schacter (2016). Lang Cogn Neurosci 32(6):669-673. https://doi.org/10.1080/23273798.2016.1252847

Davison EN, Schlesinger KJ, Bassett DS, Lynall ME, Miller MB, Grafton ST, Carlson JM (2015) Brain network adaptability across task states. PLoS Comput Biol 11(1):e1004029. https:// doi.org/10.1371/journal.pcbi.1004029

de Wit SJ, de Vries FE, van der Werf YD, Cath DC, Heslenfeld DJ, Veltman EM, van Balkom AJ, Veltman DJ, van den Heuvel OA (2012) Presupplementary motor area hyperactivity during 
response inhibition: a candidate endophenotype of obsessivecompulsive disorder. Am J Psychiatry 169(10):1100-1108. https ://doi.org/10.1176/appi.ajp.2012.12010073

Diamond A (2013) Executive functions. Annu Rev Psychol 64:135168. https://doi.org/10.1146/annurev-psych-113011-143750

Diedrichsen J, Balsters JH, Flavell J, Cussans E, Ramnani N (2009) A probabilistic MR atlas of the human cerebellum. Neuroimage 46(1):39-46. https://doi.org/10.1016/j.neuroimage.2009.01.045

Esposito R, Cieri F, Chiacchiaretta P, Cera N, Lauriola M, Di Giannantonio M, Tartaro A, Ferretti A (2018) Modifications in resting state functional anticorrelation between default mode network and dorsal attention network: comparison among young adults, healthy elders and mild cognitive impairment patients. Brain Imaging Behav 12(1):127-141. https://doi.org/10.1007/s11682-017-9686-y

Fadili MJ, Bullmore ET (2004) A comparative evaluation of waveletbased methods for hypothesis testing of brain activation maps. Neuroimage 23(3):1112-1128. https://doi.org/10.1016/j.neuro image.2004.07.034

Fan L, Li H, Zhuo J, Zhang Y, Wang J, Chen L, Yang Z, Chu C, Xie S, Laird AR, Fox PT, Eickhoff SB, Yu C, Jiang T (2016) The human brainnetome atlas: a new brain atlas based on connectional architecture. Cereb Cortex 26(8):3508-3526. https://doi. org/10.1093/cercor/bhw157

Finn ES, Shen X, Scheinost D, Rosenberg MD, Huang J, Chun MM, Papademetris X, Constable RT (2015) Functional connectome fingerprinting: identifying individuals using patterns of brain connectivity. Nat Neurosci 18(11):1664-1671. https://doi. org/10.1038/nn.4135

Fortenbaugh FC, Rothlein D, McGlinchey R, DeGutis J, Esterman M (2018) Tracking behavioral and neural fluctuations during sustained attention: a robust replication and extension. Neuroimage 171:148-164. https://doi.org/10.1016/j.neuroimage.2018.01.002

Funahashi S, Andreau JM (2013) Prefrontal cortex and neural mechanisms of executive function. J Physiol Paris 107(6):471-482. https://doi.org/10.1016/j.jphysparis.2013.05.001

Gerrits NJ, van der Werf YD, Verhoef KM, Veltman DJ, Groenewegen HJ, Berendse HW, van den Heuvel OA (2015) Compensatory fronto-parietal hyperactivation during set-shifting in unmedicated patients with Parkinson's disease. Neuropsychologia 68:107-116. https://doi.org/10.1016/j.neuropsychologi a.2014.12.022

Gratton C, Laumann TO, Nielsen AN, Greene DJ, Gordon EM, Gilmore AW, Nelson SM, Coalson RS, Snyder AZ, Schlaggar BL, Dosenbach NUF, Petersen SE (2018) Functional brain networks are dominated by stable group and individual factors, not cognitive or daily variation. Neuron 98(2):439 e435-452 e435. https:// doi.org/10.1016/j.neuron.2018.03.035

Griffa A, Baumann PS, Thiran JP, Hagmann P (2013) Structural connectomics in brain diseases. NeuroImage 80:515-526. https://doi. org/10.1016/j.neuroimage.2013.04.056

Gu S, Yang M, Medaglia JD, Gur RC, Gur RE, Satterthwaite TD, Bassett DS (2017) Functional hypergraph uncovers novel covariant structures over neurodevelopment. Hum Brain Mapp 38(8):38233835. https://doi.org/10.1002/hbm.23631

Hearne LJ, Cocchi L, Zalesky A, Mattingley JB (2017) Reconfiguration of brain network architectures between resting-state and complexity-dependent cognitive reasoning. J Neurosci 37(35):8399-8411. https://doi.org/10.1523/JNEUROSCI.0485-17.2017

Horien C, Shen X, Scheinost D, Constable RT (2019) The individual functional connectome is unique and stable over months to years. Neuroimage 189:676-687. https://doi.org/10.1016/j.neuroimage .2019.02.002

Iordan AD, Reuter-Lorenz PA (2017) Age-related change and the predictive value of the "Resting state": a commentary on Campbell and Schacter (2016). Lang Cogn Neurosci 32(6):674-677. https ://doi.org/10.1080/23273798.2016.1242759
Ito T, Kulkarni KR, Schultz DH, Mill RD, Chen RH, Solomyak LI, Cole MW (2017) Cognitive task information is transferred between brain regions via resting-state network topology. Nat Commun 8(1):1027. https://doi.org/10.1038/s41467-017-01000-w

Kaller CP, Debelak R, Kostering L, Egle J, Rahm B, Wild PS, Blettner M, Beutel ME, Unterrainer JM (2016) Assessing planning ability across the adult life span: population-representative and ageadjusted reliability estimates for the tower of London (TOL-F). Arch Clin Neuropsychol 31(2):148-164. https://doi.org/10.1093/ $\operatorname{arclin} /$ acv088

Krienen FM, Yeo BT, Buckner RL (2014) Reconfigurable task-dependent functional coupling modes cluster around a core functional architecture. Philos Trans R Soc Lond B Biol Sci. https://doi. org/10.1098/rstb.2013.0526

Langer N, Pedroni A, Gianotti LR, Hanggi J, Knoch D, Jancke L (2012) Functional brain network efficiency predicts intelligence. Hum Brain Mapp 33(6):1393-1406. https://doi.org/10.1002/hbm.21297

Latora V, Marchiori M (2001) Efficient behavior of small-world networks. Phys Rev Lett 87(19):198701. https://doi.org/10.1103/ PhysRevLett.87.198701

Lopes R, Delmaire C, Defebvre L, Moonen AJ, Duits AA, Hofman P, Leentjens AF, Dujardin K (2017) Cognitive phenotypes in parkinson's disease differ in terms of brain-network organization and connectivity. Hum Brain Mapp 38(3):1604-1621. https://doi. org/10.1002/hbm.23474

Lord LD, Stevner AB, Deco G, Kringelbach ML (2017) Understanding principles of integration and segregation using whole-brain computational connectomics: implications for neuropsychiatric disorders. Philos Trans A Math Phys Eng Sci. https://doi.org/10.1098/ rsta.2016.0283

Mak LE, Minuzzi L, MacQueen G, Hall G, Kennedy SH, Milev R (2017) The default mode network in healthy individuals: a systematic review and meta-analysis. Brain Connect 7(1):25-33. https:// doi.org/10.1089/brain.2016.0438

Matheson GJ (2019) We need to talk about reliability: making better use of test-retest studies for study design and interpretation. PeerJ 7:e6918. https://doi.org/10.7717/peerj.6918

Meijer KA, Eijlers AJC, Douw L, Uitdehaag BMJ, Barkhof F, Geurts JJG, Schoonheim MM (2017) Increased connectivity of hub networks and cognitive impairment in multiple sclerosis. Neurology 88(22):2107-2114. https://doi.org/10.1212/WNL.0000000000 003982

Miranda-Dominguez O, Mills BD, Carpenter SD, Grant KA, Kroenke CD, Nigg JT, Fair DA (2014) Connectotyping: model based fingerprinting of the functional connectome. PLoS ONE 9(11):e111048. https://doi.org/10.1371/journal.pone.0111048

Nichols TE, Das S, Eickhoff SB, Evans AC, Glatard T, Hanke M, Kriegeskorte N, Milham MP, Poldrack RA, Poline JB, Proal E, Thirion B, Van Essen DC, White T, Yeo BT (2017) Best practices in data analysis and sharing in neuroimaging using MRI. Nat Neurosci 20(3):299-303. https://doi.org/10.1038/nn.4500

Noble S, Scheinost D, Constable RT (2019) A decade of test-retest reliability of functional connectivity: a systematic review and metaanalysis. Neuroimage 203:116157. https://doi.org/10.1016/j.neuro image.2019.116157

Nowrangi MA, Lyketsos C, Rao V, Munro CA (2014) Systematic review of neuroimaging correlates of executive functioning: converging evidence from different clinical populations. J Neuropsychiatry Clin Neurosci 26(2):114-125. https://doi.org/10.1176/ appi.neuropsych.12070176

Oldfield RC (1971) The assessment and analysis of handedness: the Edinburgh inventory. Neuropsychologia 9(1):97-113

Owen AM (1997) Cognitive planning in humans: neuropsychological, neuroanatomical and neuropharmacological perspectives. Prog Neurobiol 53(4):431-450 
Parkes L, Fulcher B, Yucel M, Fornito A (2018) An evaluation of the efficacy, reliability, and sensitivity of motion correction strategies for resting-state functional MRI. Neuroimage 171:415-436. https ://doi.org/10.1016/j.neuroimage.2017.12.073

Patenaude B, Smith SM, Kennedy DN, Jenkinson M (2011) A Bayesian model of shape and appearance for subcortical brain segmentation. Neuroimage 56(3):907-922. https://doi.org/10.1016/j.neuro image.2011.02.046

Phillips LH, Wynn VE, McPherson S, Gilhooly KJ (2001) Mental planning and the Tower of London task. Q J Exp Psychol A 54(2):579-597. https://doi.org/10.1080/713755977

Power JD, Schlaggar BL, Petersen SE (2015) Recent progress and outstanding issues in motion correction in resting state fMRI. Neuroimage 105:536-551. https://doi.org/10.1016/j.neuroimage .2014 .10 .044

Pruim RHR, Mennes M, van Rooij D, Llera A, Buitelaar JK, Beckmann CF (2015) ICA-AROMA: A robust ICA-based strategy for removing motion artifacts from fMRI data. Neuroimage 112:267-277. https://doi.org/10.1016/j.neuroimage.2015.02.064

Putcha D, Ross RS, Cronin-Golomb A, Janes AC, Stern CE (2016) Salience and default mode network coupling predicts cognition in aging and Parkinson's disease. J Int Neuropsychol Soc 22(2):205215. https://doi.org/10.1017/S1355617715000892

Rabinovici GD, Stephens ML, Possin KL (2015) Executive dysfunction. Continuum (Minneap Minn) 21:646-659. https://doi. org/10.1212/01.CON.0000466658.05156.54(3 Behavioral Neurology and Neuropsychiatry)

Rocca MA, Valsasina P, Meani A, Falini A, Comi G, Filippi M (2016) Impaired functional integration in multiple sclerosis: a graph theory study. Brain Struct Funct 221(1):115-131. https://doi. org/10.1007/s00429-014-0896-4

Rubinov M, Sporns O (2010) Complex network measures of brain connectivity: uses and interpretations. Neuroimage 52(3):1059-1069. https://doi.org/10.1016/j.neuroimage.2009.10.003

Schmand BA, Bakker D, Saan RJ, Louman J (1991) De Nederlandse Leestest voor Volwassenen: een maat voor het premorbide intelligentieniveau. Tijdschr Gerontol Geriatr 22:15-19

Shallice T (1982) Specific impairments of planning. Philos Trans R Soc Lond B Biol Sci 298(1089):199-209. https://doi.org/10.1098/ rstb.1982.0082

Sheffield JM, Repovs G, Harms MP, Carter CS, Gold JM, MacDonald AW 3rd, Daniel Ragland J, Silverstein SM, Godwin D, Barch DM (2015) Fronto-parietal and cingulo-opercular network integrity and cognition in health and schizophrenia. Neuropsychologia 73:82-93. https://doi.org/10.1016/j.neuropsychologi a.2015.05.006

Sheffield JM, Kandala S, Tamminga CA, Pearlson GD, Keshavan MS, Sweeney JA, Clementz BA, Lerman-Sinkoff DB, Hill SK, Barch DM (2017) Transdiagnostic associations between functional brain network integrity and cognition. JAMA Psychiatry 74(6):605613. https://doi.org/10.1001/jamapsychiatry.2017.0669

Shine JM, Poldrack RA (2018) Principles of dynamic network reconfiguration across diverse brain states. Neuroimage 180(Pt B):396405. https://doi.org/10.1016/j.neuroimage.2017.08.010

Shine JM, Bissett PG, Bell PT, Koyejo O, Balsters JH, Gorgolewski KJ, Moodie CA, Poldrack RA (2016) The dynamics of functional brain networks: integrated network states during cognitive task performance. Neuron 92(2):544-554. https://doi.org/10.1016/j. neuron.2016.09.018

Smith SM, Jenkinson M, Woolrich MW, Beckmann CF, Behrens TE, Johansen-Berg H, Bannister PR, De Luca M, Drobnjak I, Flitney DE, Niazy RK, Saunders J, Vickers J, Zhang Y, De Stefano N, Brady JM, Matthews PM (2004) Advances in functional and structural MR image analysis and implementation as FSL. Neuroimage 23(Suppl 1):S208-219. https://doi.org/10.1016/j.neuro image.2004.07.051

Stanley ML, Simpson SL, Dagenbach D, Lyday RG, Burdette JH, Laurienti PJ (2015) Changes in brain network efficiency and working memory performance in aging. PLoS ONE 10(4):e0123950. https ://doi.org/10.1371/journal.pone.0123950

Telesford QK, Lynall ME, Vettel J, Miller MB, Grafton ST, Bassett DS (2016) Detection of functional brain network reconfiguration during task-driven cognitive states. NeuroImage 142:198-210. https ://doi.org/10.1016/j.neuroimage.2016.05.078

van den Heuvel OA, Groenewegen HJ, Barkhof F, Lazeron RH, van Dyck R, Veltman DJ (2003) Frontostriatal system in planning complexity: a parametric functional magnetic resonance version of Tower of London task. Neuroimage 18(2):367-374

van den Heuvel MP, Stam CJ, Kahn RS, Hulshoff Pol HE (2009) Efficiency of functional brain networks and intellectual performance. J Neurosci 29(23):7619-7624. https://doi.org/10.1523/JNEUR OSCI.1443-09.2009

Verhage F (1964) Intelligentie en leeftijd: Onderzoek bij Nederlands van twaal tot zevenenzeventig jaar. van Gorcum, Assen

Wang J, Zuo X, He Y (2010) Graph-based network analysis of resting-state functional MRI. Front Syst Neurosci 4:16. https://doi. org/10.3389/fnsys.2010.00016

Welton T, Kent DA, Auer DP, Dineen RA (2015) Reproducibility of graph-theoretic brain network metrics: a systematic review. Brain Connect 5(4):193-202. https://doi.org/10.1089/brain.2014.0313

Worbe Y (2015) Neuroimaging signature of neuropsychiatric disorders. Curr Opin Neurol 28(4):358-364. https://doi.org/10.1097/ WCO.0000000000000220

Yeo BT, Krienen FM, Sepulcre J, Sabuncu MR, Lashkari D, Hollinshead M, Roffman JL, Smoller JW, Zollei L, Polimeni JR, Fischl B, Liu H, Buckner RL (2011) The organization of the human cerebral cortex estimated by intrinsic functional connectivity. J Neurophysiol 106(3):1125-1165. https://doi.org/10.1152/jn.00338.2011

Zhang Z, Telesford QK, Giusti C, Lim KO, Bassett DS (2016) Choosing wavelet methods, filters, and lengths for functional brain network construction. PLoS ONE 11(6):e0157243. https://doi. org/10.1371/journal.pone.0157243

Zuo XN, Xu T, Milham MP (2019) Harnessing reliability for neuroscience research. Nat Hum Behav 3(8):768-771. https://doi. org/10.1038/s41562-019-0655-x

Publisher's Note Springer Nature remains neutral with regard to jurisdictional claims in published maps and institutional affiliations. 